\title{
Patient-derived Xenografts from Colorectal Carcinoma: A Temporal and Hierarchical Study of Murine Stromal Cell Replacement
}

\author{
CELIA CHAO $^{1}$, STEVE G. WIDEN ${ }^{2}$, THOMAS G. WOOD ${ }^{2}$, JOHN R. ZATARAIN ${ }^{1}$, \\ PAUL JOHNSON ${ }^{1}$, AAKASH GAJJAR ${ }^{1}$, GUILLERMO GOMEZ ${ }^{1}$, SUIMIN QIU $^{3}$, \\ JILL THOMPSON $^{2}$, HEIDI SPRATT ${ }^{4}$ and MARK R. HELLMICH ${ }^{1}$ \\ Departments of ${ }^{1}$ Surgery, ${ }^{2}$ Biochemistry and Molecular Biology, \\ ${ }^{3}$ Surgical Pathology and ${ }^{4}$ Preventative Medicine and Community Health \\ University of Texas Medical Branch, Galveston, TX, U.S.A.
}

\begin{abstract}
Background/Aim: Patient-derived xenografting (PDX) of human colorectal cancer (CRC) is the preferred experimental model to study tumor response to therapeutic agents. Gradually, human stromal cells are replaced by mouse stromal cells; however, the exact timing of the replacement of human with murine stromal cells in human CRC xenograft has not been fully elucidated. We hypothesize that orthologous murine transcripts functionally substitutes for the loss due to replacement of human stromal genes. Materials and Methods: Human CRC were implanted in athymic nude mice in replicates and followed-up over time. Using next-generation sequencing, we determined the temporal kinetics of human stromal cell replacement with the orthologous murine transcripts. Results: CRC cell-induced re-organization of the normal, quiescent murine stromal cells into a protumorigenic phenotype supporting human CRC growth occurs at initial implantation. Conclusion: Murine cell replacement occurs in a time- and size-dependent manner.
\end{abstract}

Patient-derived xenografts (PDX) have emerged as a superior experimental model to study tumor response to therapeutic agents (1). As a pre-clinical platform for incipient drug testing, the PDX has greater predictive value compared to xenografts generated from human cancer cell lines injected into

This article is freely accessible online.

Correspondence to: Dr. Celia Chao, Department of Surgery, University of Texas Medical Branch, 301 University Blvd., Galveston, 77555 TX, U.S.A. Tel: +1 4097720698, e-mail: cechao@utmb.edu

Key Words: Patient derived xenografts, human colorectal carcinoma, next-generation sequencing. immunocompromised mice (2). To model human colorectal cancer (CRC), PDX has been shown to exhibit the authentic characteristics of the patient's tumor tissue, including maintenance of histopathological architecture, preservation of the human cancer gene transcripts, intratumoral clonal heterogeneity, and chromosomal instability (3-5).

In contrast to xenografts derived from established CRC cell lines, the PDX model involves transplanting whole human CRC tissue, which includes not only the malignant cells but also the peritumoral stromal cells within the patient's tumor. A small piece of human whole-tumor tissue is directly implanted into immunocompromised mice. Over time, the human stromal cells are replaced by mouse stromal cells (68); however, the exact timing and replacement of human with murine stromal cells in human CRC is not clear (9). The loss of human cancer-associated fibroblasts, endothelial cells, and immune cells over time has been characterized as a pitfall of the PDX model because mixed murine and human receptorligand signaling may alter the human cancer phenotype (9-11). However, in a recent report by Isella et al., the murine stromal transcripts derived from CRC PDXs recapitulated the prognostic mesenchymal gene signature of human CRC tumors (12). These findings indicate the stromal-derived transcripts have prognostic and predictive significance, not the cancer cell transcripts and that the CRC PDX overwhelmingly models appropriate reciprocal paracrine signaling between the cancer cell and the murine stromal cells.

We hypothesize that the replacement by orthologous murine transcripts functionally substitutes for the loss, due to replacement of human stromal genes, indicating that the cancer cells have necessarily acquired and activated the resident murine stromal cells to promote CRC tumor growth in the PDX. In this report, we implanted human CRC in athymic nude mice in replicates and followed-up over time the orthologous murine transcripts that replace the human 
stromal gene transcripts in order to determine the temporal kinetics of human stromal cell replacement.

\section{Materials and Methods}

Colorectal cancer specimens. Freshly resected colorectal cancer tissue was collected under an IRB-approved protocol, dissected by a surgical pathologist, and immediately placed into a sterile tube containing cold DMEM supplemented with $200 \mathrm{U} / \mathrm{ml}$ penicillin, $200 \mu \mathrm{g} / \mathrm{ml}$ streptomycin, $0.25 \mu \mathrm{g} / \mathrm{ml}$ amphotericin $\mathrm{B}$, and $50 \mu \mathrm{g} / \mathrm{ml}$ gentamicin (Invitrogen; Carlsbad, CA, USA). After several washings in this medium, a small aliquot is preserved in RNAlater ${ }^{\circledR}$ (ThermoFisher Scientific; Waltham, MA, USA), another small piece of tumor is fixed in $10 \%$ formalin, and the final portion is minced into $2-3 \mathrm{~mm}$ diameter pieces under sterile conditions for engraftment.

Mice. Mice are housed in our barrier- and virus antibody-free housing. Under an IACUC-approved protocol, three- to nine-monthold male or female athymic nude mice (Envigo Laboratories; Indianapolis, IN) are placed under isoflurane anesthesia, and the tumor fragments are engrafted into a subcutaneous pocket in the mice under sterile conditions. Mice received Buprenorphine SR $(1 \mathrm{mg} / \mathrm{kg}$ SQ) immediately post-procedure. The mice were monitored for tumor take and were sacrificed at the specified intervals based on transcutaneous tumor size. At sacrifice, a small portion of the xenografted tumor tissue was flash frozen in liquid nitrogen and subjected to RNA extraction at a later date; another small piece was fixed in $10 \%$ neutral formalin and embedded in paraffin for histological analyses; and the remainder was minced and transferred into cryopreserving solution for future passage in mice or directly passed into mice as the following generation.

PDX tumors. Under an IRB-approved protocol, primary human colorectal cancers were freshly prosected in the surgical pathology suite. For the temporal experiments, the $2-3 \mathrm{~mm}$ diameter pieces of patient tumor were implanted in triplicate into athymic nude mice. Mice were sacrificed serially when the tumor was approximately 4-6 mm (early), 8-12 $\mathrm{mm}$ (intermediate), and 16-20 $\mathrm{mm}$ in largest diameter (late) (Figure 1). PDX tumors originating from initial xenografting of the human CRC tissue is termed Passage 0 (P0). PDX tumors originating from a P0 tumor is termed Passage 1 (P1) and so forth.

Library construction and sequencing. Total RNA was isolated using an RNAqueous protocol (LifeTech) and RNA quality was assessed using an RNA 6000 chip on an Agilent 2100 Bioanalyzer (Agilent Technologies). Poly A+ RNA was selected and libraries were prepared with the TruSeq RNA Library Preparation kit v2 (Illumina) following the manufacturer's recommended protocol. Paired-end 50 base sequencing was performed using an Illumina HiSeq 1500. All libraries were indexed; 6 libraries per lane were loaded to get at least 25-30 million read-pairs per sample. Paired-end reads can help reduce uncertainty in mapping reads and will help in discriminating between mouse and human (13).

NGS run quality assessment. Quality was evaluated initially by Illumina's internal assessment of each run. Overall run quality per lane and per cycle was assessed. After demultiplexing the samples, FASTQC (14) was used to provide per sample information on base percentage distribution per cycle, GC content across the run, percent uncalled bases, and percent duplication of sequences.
RNA-Seq analysis. The alignment of NGS sequence reads was performed using the Spliced Transcript Alignment to a Reference (STAR) program (15). We have created and used a combined reference with the human hg38 and mouse mm10 assemblies. Mapping the reads to the combined reference allows the mapping program to find the best match between the human and mouse (16). Only reads mapping unambiguously were counted using the featureCounts utility of the subread analysis software (17).

Gene ontology and pathway analysis was performed using Gene Set Enrichment Analysis (GSEA) (18-20). Human stromal and immune signature gene lists were taken from Yoshihara et al. and Isella et al. and expression levels from all signature genes with mouse orthologs were selected $(12,21)$. The $\mathrm{R}$ package gplots was used to create the heatmaps (22).

\section{Results}

Subcutaneous (SQ) implantation of human CRC recapitulates the histopathologic architecture of the original tumor. In contrast to xenografts derived from established CRC cell lines, the PDX model involves transplanting whole human CRC tissue, which includes not only the malignant cells but also the activated stroma of the patient's tumor in the correct spatial orientation. PDXs exhibit the authentic characteristics of the patient's tumor tissue from which they were derived, including maintenance of histopathological architecture, preservation of the human cancer gene transcripts as the tumor tissue is serially passaged in mice, and tumor response to therapeutic agents $(5,23)$. We confirm that xenografting intact human CRC tissue in athymic nude mice results in the maintenance of the original morphological features that histologically characterize the original human tumor resected from the patient (Figure 2). Remarkably, the principal features of the parent tumor architecture are preserved during sequential passages from the first piece of tumor (Passage Zero, P0, Figure 1) into subsequent early passages (P2). Notably, the specific organization of the epithelial and stromal cells in relation to each other was recapitulated in the PDX tumors. These data suggest that the implanted human cancer cells instruct normal mouse stromal cells to organize spatially and functionally to support human cancer cell growth in a unique patient tumor-specific manner.

Human CRC cells maintain the same molecular capabilities to interact with and instruct the murine stromal cells. To evaluate the contribution of human versus murine transcriptomes in the xenografts, we performed Next Generation Sequencing (RNA-Seq) on 10 primary human CRC and their corresponding P0, P1, P2, or P4 xenografts, and performed species-specific molecular characterization of the expression data. The reads from each library, both the original tumor and corresponding xenograft, were mapped to a combined genome reference sequence consisting of the human (hg38) and the mouse (mm10) reference genomes. Only reads mapping unambiguously to one or the other were 


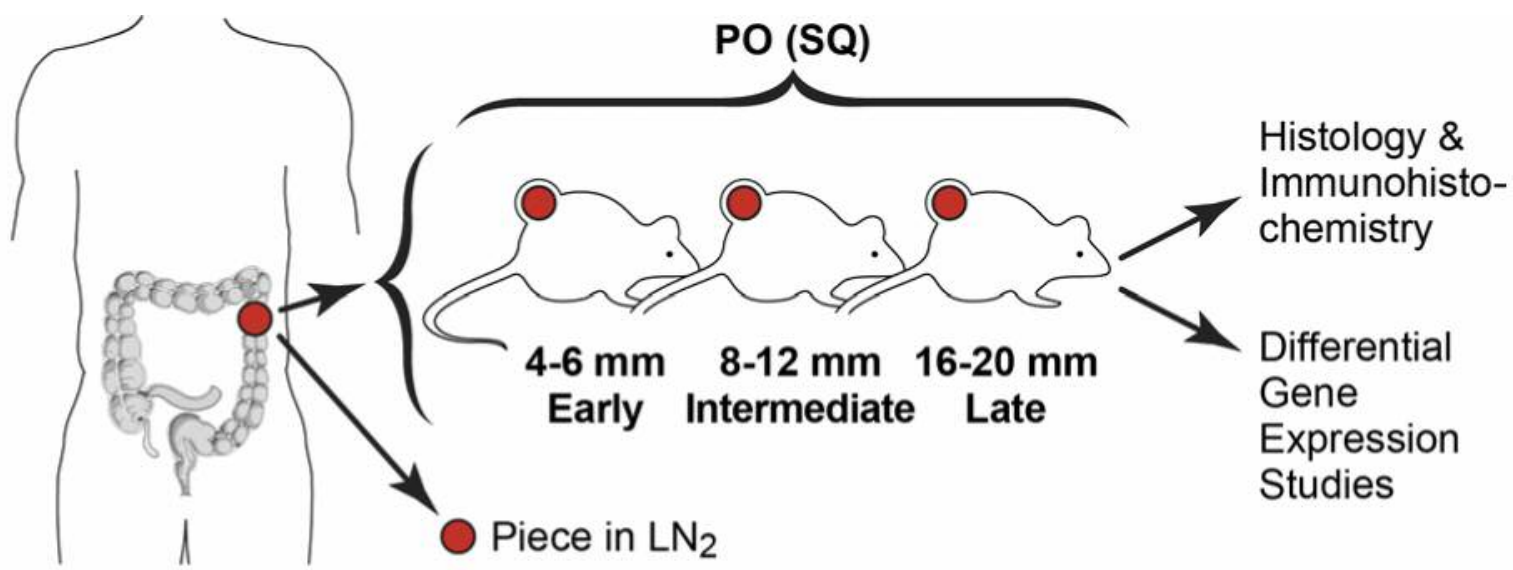

Figure 1. Defining time- and size-dependent replacement of human transcripts (hg38) by orthologous murine transcripts (mm10) in Passage Zero (PO) human CRC xenografts.
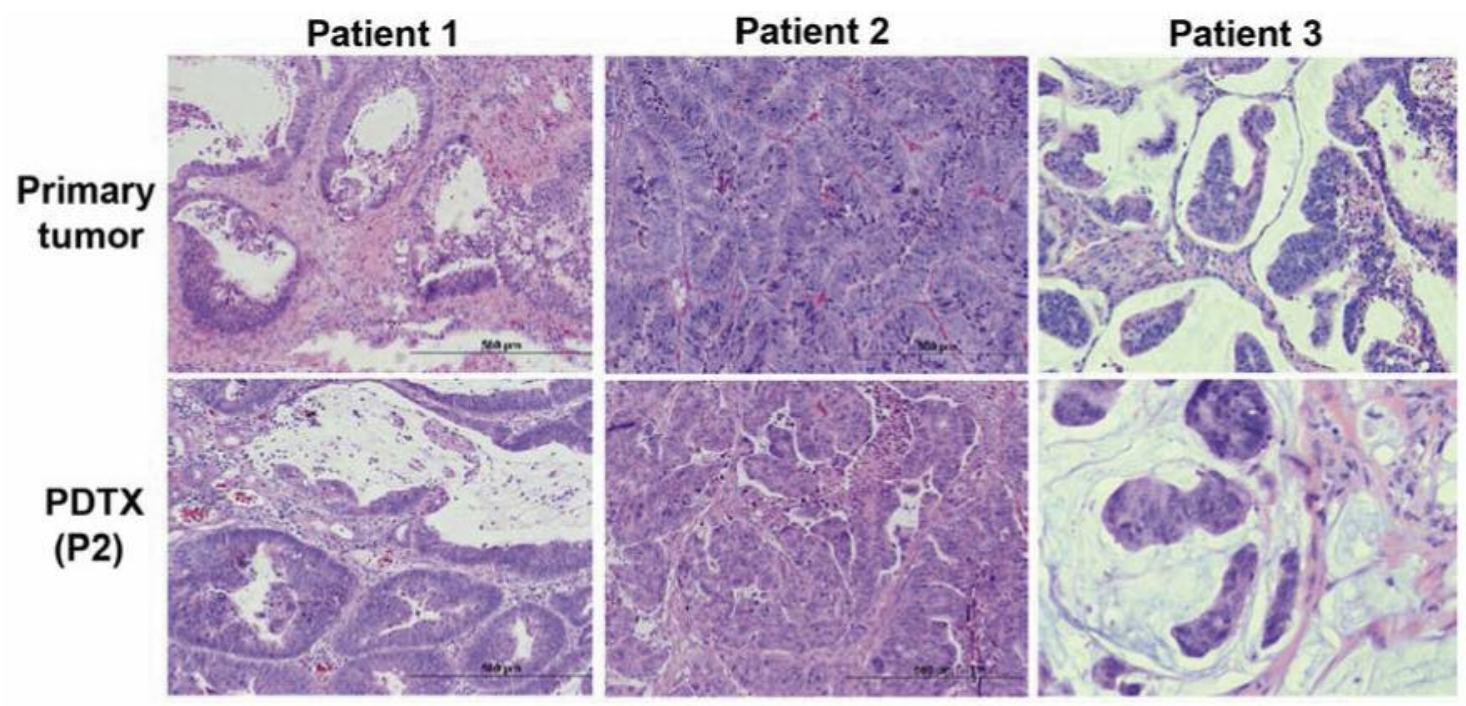

Figure 2. Hematoxylin and eosin stained sections of original CRC from three representative patients. The histopathology of Patients 1 and 3 show mucin-filled ductal structures, whereas the tumor from Patient 2 is densely cellular. P2=Passage 2 .

counted. Across all 10 tumor sets evaluated, the human transcripts present in the parent tumor were maintained in the corresponding P0-4 xenograft with Pearson's correlation coefficient of $\sim 0.9$ (range $=0.87-0.96$; Table I). Comparison of the human transcripts from the $\mathrm{P} 0 v s$. $\mathrm{P} 1$ and $\mathrm{P} 2 v s$. P4 (between early passage PDXs) show $\geq 0.97$ Pearson's correlation coefficient (Table I, column 2). These data indicate that the xenografts maintain the cancer cell transcriptomes with very high fidelity in early passage PDX.

Orthologous murine stromal genes substitute for relevant human stromal genes to support human CRC xenograft growth at PO in a tumor mass-dependent manner. When comparing the human transcripts from the original patient's and xenograft tumors, a small fraction of human stromal transcripts common among all ten tumors were lost in the early (P0-P4) passage PDX (Table I), and replaced by orthologous murine stromal transcripts. Since a detailed determination of the temporal and hierarchical activation of the murine stromal transcripts that support the early PDX tumors had not been performed previously, we mapped the kinetics of human cancer cell-directed stromal activation at the tissue level by comparing P0 early (4-6 mm diameter tumor size), intermediate $(8-12 \mathrm{~mm})$, and late $(16-20 \mathrm{~mm})$ 


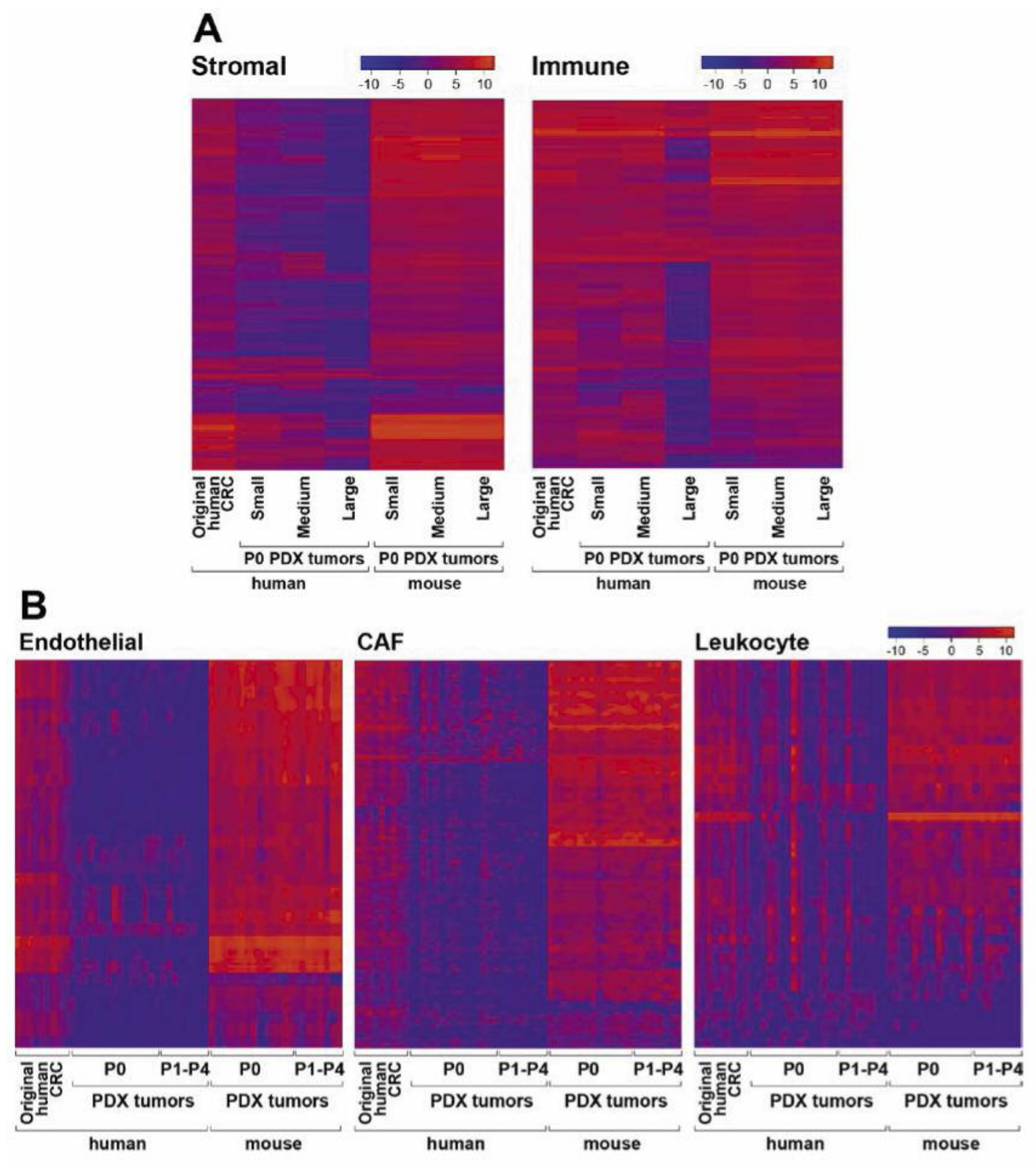

Figure 3. A. Heat maps matching lost human and gained murine transcripts in the PDX to published human cancer stromal and immune signatures (21). The log2 average of transcripts per million (TPM) are shown. B. Heat Map comparing human genes (left) or mouse genes (right) in ten original human CRCs and their corresponding P0, P1-4 PDX tumors. Gene list from the three major components of the tumor stroma (CAF, endothelial, and leukocyte) with prognostic value (12). The log2 of transcripts per million (TPM) are shown.

xenograft transcriptome profiles (replaced murine stromal transcripts) (Figure 1). The time to reach the tumor size (early, intermediate, and late) varied by the individual CRC tumor implanted. Some tumor implants grew subcutaneous nodules within 3-4 weeks while other tumors had a long lag time of 6-8 months prior to developing a measurable subcutaneous mass.
To directly compare the human to murine transcript replacement process over time, we mapped the human and mouse transcripts to two published databases: 1) ESTIMATE, a computational method using gene expression signatures that infers the fraction of human stromal and immune cells in tumors (21) (Figures 2 and 3A) a PDX stromal signature with prognostic significance in human 


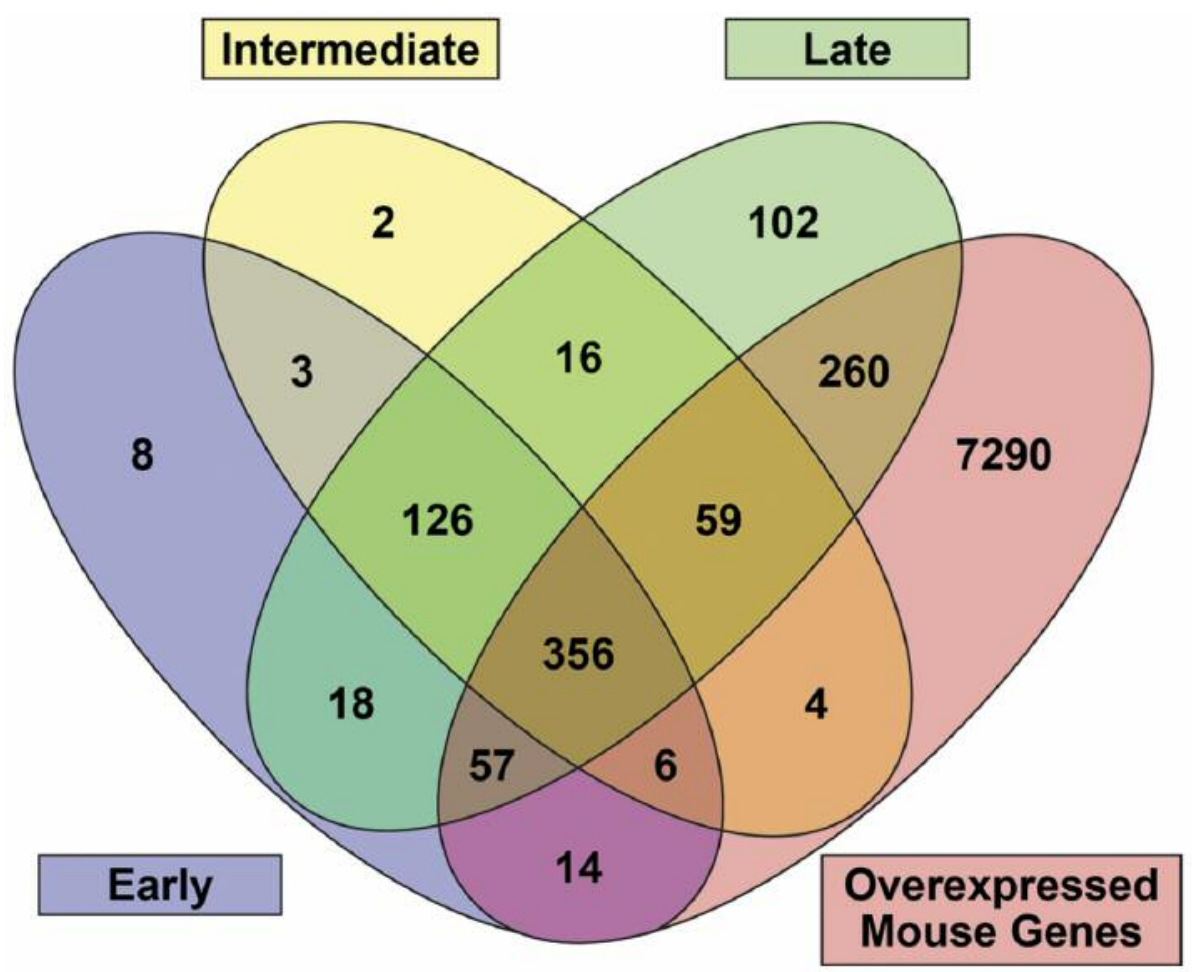

Figure 4. Venn diagram demonstrating overlap of human transcripts in the early, intermediate and late PDX tumors which were decreased significantly at least 4-fold compared to the original human CRC tumor.

Table I. Comparison of concordant human and mouse transcripts between two samples.

\begin{tabular}{|c|c|c|}
\hline CRC patient & $\begin{array}{l}\text { Pearson's Correlation Coefficient: } \\
\text { Human transcripts }\end{array}$ & $\begin{array}{l}\text { Pearson's Correlation Coefficient: } \\
\text { Mouse transcripts }\end{array}$ \\
\hline 1 & 0.95 (original human $\mathrm{CRC} v s . \mathrm{P} 2$ ) & N/A \\
\hline 2 & 0.95 (original human CRC vs. P2) & N/A \\
\hline \multirow[t]{2}{*}{3} & 0.91 (original human CRC vs. P2) & \\
\hline & 0.99 (P2 vs. P4) & 0.94 (P2 vs. P4) \\
\hline \multirow[t]{2}{*}{4} & 0.92 (original human CRC vs. large P0) & \\
\hline & 0.91 (original human CRC vs. P1) & 0.97 (large P0 vs. P1) \\
\hline \multirow[t]{2}{*}{5} & 0.90 (original human CRC vs. large $\mathrm{P} 0$ ) & \\
\hline & 0.89 (original human CRC vs. P1) & 0.96 (large P0 vs. P1) \\
\hline \multirow[t]{2}{*}{6} & 0.96 (original human CRC vs. large P0) & \\
\hline & $0.98(\mathrm{P} 0$ vs. $\mathrm{P} 1)$ & 0.96 (large P0 vs. P1) \\
\hline \multirow[t]{3}{*}{7} & 0.90 (original human CRC vs. small P0) & \\
\hline & 0.90 (original human CRC vs. medium P0) & 0.92 (small vs. medium $\mathrm{P} 0)$ \\
\hline & 0.87 (original human CRC vs. large $\mathrm{P} 0$ ) & 0.94 (medium vs. large $\mathrm{P} 0$ ) \\
\hline \multirow[t]{4}{*}{8} & 0.95 (original human tumor vs. small P0) & 0.99 (large P0 vs. P1) \\
\hline & 0.94 (original human tumor $v s$. medium $\mathrm{P} 0$ ) & 0.92 (small vs. medium) \\
\hline & 0.92 (original human tumor $v s$. large $\mathrm{P} 0$ ) & 0.92 (medium vs. large) \\
\hline & 0.92 (original human tumor $v s . \mathrm{P} 1$ ) & 0.96 (large P0 vs. P1) \\
\hline \multirow[t]{2}{*}{9} & 0.94 (original human tumor $v s$. small $\mathrm{P} 0$ ) & \\
\hline & 0.91 (original human tumor $v s$. medium $\mathrm{P} 0$ ) & 0.94 (small vs. medium P0) \\
\hline \multirow[t]{3}{*}{10} & 0.95 (original human tumor $v s$. small $\mathrm{P} 0$ ) & \\
\hline & 0.93 (original human tumor $v s$. med $\mathrm{P} 0$ ) & 0.97 (small vs. medium P0) \\
\hline & 0.92 (original human tumor $v s$. large $\mathrm{P} 0$ ) & 0.98 (medium $v s$. large $\mathrm{P} 0$ ) \\
\hline
\end{tabular}

P0: Original human tumor xenografted into immunocompromised mouse at first implantation; P1: P0 tumor xenografted at next passage. 
CRC tumors (12) (Figure 3B). Figures 3A and 3B show the human stromal signature transcripts that were decreased (blue) in the PDX relative to the original patient tumors, and matched to corresponding orthologous murine transcripts that were enriched (red) in the xenografts relative to the human tumors. A 16-fold difference is illustrated in the color scale as a $\log 2$ fold-change of -4 and +4 . The exchange of mouse stroma for human stroma (blood vessel cells, fibroblasts, and immune cells) occurs early in the smallest tumor (4-6 mm, Figure 1). The murine genes are gained (red) immediately within the smallest tumors and persist as the tumor grows larger. Complete replacement of the human endothelial cell-related transcripts by the murine orthologs occurs the most rapidly, whereas the human fibroblast and human immune transcripts persist longer, present in the medium and large PDX tumors.

The human stromal cells in the PDX model are replaced by murine stromal transcripts which remain stable at early passages. The murine transcripts remain stable at early PDX passages (P0-P4), with Pearson's coefficients $\geq 0.94$ (Table I, column 3); conservation of the cancer cell transcriptomes in early passage PDX indicates that the passaged cancer cells retain the same molecular capabilities to reprogram the murine cells of the tumor microenvironment with remarkable stability between early passages.

A Venn diagram of the relative gain of murine stromal genes (and concomitant loss of human orthologous genes) by tumor size is shown in Figure 4. There are 356 (35\%) human genes at least 4-fold significantly decreased from the original human CRC tumor (increased murine orthologs in the PDX) that overlap between the early, intermediate, and late P0 tumors, indicating that at least a third of the influx of murine transcripts occurs immediately in the smallest size PDX tumor. As the tumors grow and develop larger mass, additional murine-derived transcripts at least 4-fold increased overlap only between the intemediate and late tumors $(7 \%)$. Approximately $35 \%$ of murine transcript at least 4-fold inreased are found only in the large P0 PDX tumors. As the PDX mass increases, the stromal human transcripts become diluted to a smaller percentage of the total transcripts.

Gene Set Enrichment Analysis (GSEA) of the human transcripts which lost expression in the 10 PDX relative to the parent human CRC tumor showed that among the Molecular Signatures Databases v6.0 interrogated, the top two hallmark gene sets among the early, intermediate, and late tumors are Epithelial-mesenchymal transition and Inflammatory response consistent with the heat maps shown in Figure 3. Other top 10 hallmark gene sets common between the three tumors sizes include TNFA Signaling via NFkB, KRAS Signaling Up, Coagulation, and Complement (Innate Immunity). The early and intermediate, but not the late, tumors listed Angiogenesis among the top 10 hallmark gene set, whereas the only the intermediate tumors had Hypoxia as a top 10 hallmark gene set. Top KEGG and Reactome gene sets for all three tumor sizes include: Focal adhesion, ECM receptor interaction, Cytokine-cytokine receptor interaction, ECM organization, Collagen formation, and Hemostasis.

\section{Discussion}

In addition to cell-autonomous mechanisms, cancer cells activate (reprogram) their surrounding stromal cells to promote their survival, growth, and spread (24). The temporal and hierarchical development of the implanted PDX with regard to how the transformed epithelial cells and the non-transformed replacement stromal cells globally engage in reciprocal signaling networks to promote tumor growth have not been fully elucidated. Here, we show that the stromal-derived transcripts from the original human tumor specimen are lost at initial implantation, but replaced by the orthologous murine transcripts at initial implantation. The kinetics of replacement is proportional to the tumor mass; the larger the tumor, the more murine stromal genes are present to support the growing PDX. The replacement by murine orthologous transcripts recapitulates the human stromal signature of the original CRC tumor. The human non-transformed endothelial, fibroblasts, and immune cells cannot replicate and are replaced by their murine counterparts. The murine stromal cells are recruited to the human CRC tumor site where they are instructed by the human CRC cells to develop a tumor-promoting phenotype which functionally supports the proliferating human CRC cells. Thus, the growth of the xenograft is supported very early on by a dynamic murine tumor microenvironment that has been activated by the human cancer cells, and as it grows, the stroma continuously turns over (7).

Prior studies $(5,7,8,25)$ suggested that the time frame for total murine stroma replacement occurs early after implantation. A study showed that the human blood vessels supporting one human CRC xenograft growth was were replaced by the host murine cells by $\sim 3$ weeks postimplantation (26); another study suggested that murine endothelial cells completely supported the growth of the PDX by 30 days post-implantation (8). Maykel et al. reported that human stromal cells do not proliferate, determined by the absence of proliferating cell nuclear antigen (PCNA) immunostaining in the stromal compartment of PDX tumors (7). Their work suggested that the time frame for total murine replacement of human fibroblasts, immune cells, and blood vessels might occur between P0-P2. Finally, Chou (25) injected human CRC tumor cell suspensions (rather than intact tissue) and found that murine stromal cells completely replaced the human stromal cells by Passage 1 (P1). Our work firmly establishes that murine stromal cells 
are gained at P0 (initial implantation) and the increase in murine cells are proportional to the tumor mass.

Our data suggest that the different stromal microenvironmental cell types coordinately engage in reciprocal signaling with the colorectal cancer cells to provide the necessary pro-tumorigenic microenvironment to facilitate the progression of a specific PDX. Mapping the temporal transition of activated human to activated murine cancer-associated stroma shows that the transcriptomes related to angiogenesis are turned over first, followed by the transcriptomes from carcinoma-associated fibroblasts, and finally, from the immune cells. The immune cell heat map (Figure 3A) and Leukocyte heat map (Figure 3B) demonstrate that the athymic nude mouse model does replace human immune cells to support tumor growth. The mice have innate immunity (natural killer cells, B lymphocytes, and complement activity), and lack only Tcell immunity. Isella et al. recently classified CRC into three intrinsic molecular subtypes; they showed that the stem cell/serrated/mesenchymal (SSM) subtype was associated with the highest stromal content, compared to the other subtypes using the PDX model with murine stromal cell replacement analysis (12). They defined three prognostic and predictive signatures, derived from only CRC stromal cells: carcinoma-associated fibroblasts (CAF), leukocytes, and endothelial cells. Importantly, the leukocyte score was strongly correlated with microsatellite unstable CRC, whereas the CAF score correlated with poor prognosis (12).

Together, these data support the hypothesis that the normal mouse stromal cells are activated/instructed by the human cancer cells to support its own growth in a manner that the mouse stromal cells functionally substitute for the human stromal cells. CRC cell-induced re-organization of the resident, normal, quiescent murine stroma into an activated phenotype which supports human CRC growth occurs at P0 and replacement occurred in the time- and sizedependent manner.

\section{Acknowledgements}

This work was supported by Department of Surgery Bridging Grant 2014-667, John Sealy Memorial Endowment Fund, National Institutes of Health (T32DK007639, UL1TR001439, K08CA125209).

\section{References}

1 Ledford H: US cancer institute to overhaul tumour cell lines. Nature 530: 391, 2016.

2 Tentler JJ, Tan AC, Weekes CD, Jimeno A, Leong S, Pitts TM, Arcaroli JJ, Messersmith WA and Eckhardt SG: Patient-derived tumour xenografts as models for oncology drug development. Nat Rev Clin Oncol 9: 338-350, 2012.

3 Bardelli A, Corso S, Bertotti A, Hobor S, Valtorta E, Siravegna G, Sartore-Bianchi A, Scala E, Cassingena A, Zecchin D,
Apicella M, Migliardi G, Galimi F, Lauricella C, Zanon C, Perera T, Veronese S, Corti G, Amatu A, Gambacorta M, Diaz LA Jr., Sausen M, Velculescu VE, Comoglio P, Trusolino L, Di Nicolantonio F, Giordano S and Siena S: Amplification of the MET receptor drives resistance to anti-EGFR therapies in colorectal cancer. Cancer Discov 3: 658-673, 2013.

4 Guenot D, Guerin E, Aguillon-Romain S, Pencreach E, Schneider A, Neuville A, Chenard MP, Duluc I, Du Manoir S, Brigand C, Oudet P, Kedinger M and Gaub MP: Primary tumour genetic alterations and intra-tumoral heterogeneity are maintained in xenografts of human colon cancers showing chromosome instability. J Pathol 208: 643-652, 2006.

5 Julien S, Merino-Trigo A, Lacroix L, Pocard M, Goere D, Mariani P, Landron S, Bigot L, Nemati F, Dartigues P, Weiswald LB, Lantuas D, Morgand L, Pham E, Gonin P, Dangles-Marie V, Job B, Dessen P, Bruno A, Pierre A, De The H, Soliman H, Nunes M, Lardier G, Calvet L, Demers B, Prevost G, Vrignaud P, RomanRoman S, Duchamp O and Berthet C: Characterization of a large panel of patient-derived tumor xenografts representing the clinical heterogeneity of human colorectal cancer. Clin Cancer Res 18: 5314-5328, 2012.

6 Cho SY, Kang W, Han JY, Min S, Kang J, Lee A, Kwon JY, Lee C and Park H: An integrative approach to precision cancer medicine using patient-derived xenografts. Mol Cells 39: 77-86, 2016.

7 Maykel J, Liu JH, Li H, Shultz LD, Greiner DL and Houghton J: NOD-scidIl2rg (tm1Wjl) and NOD-Rag1 (null) Il2rg (tm1Wjl) : a model for stromal cell-tumor cell interaction for human colon cancer. Dig Dis Sci 59: 1169-1179, 2014.

8 Sanz L, Cuesta AM, Salas C, Corbacho C, Bellas C and AlvarezVallina L: Differential transplantability of human endothelial cells in colorectal cancer and renal cell carcinoma primary xenografts. Lab Invest 89: 91-97, 2009.

9 Rosfjord E, Lucas J, Li G and Gerber HP: Advances in patientderived tumor xenografts: from target identification to predicting clinical response rates in oncology. Biochem Pharmacol 91: 135$143,2014$.

10 Kopetz S, Lemos R and Powis G: The promise of patientderived xenografts: the best laid plans of mice and men. Clin Cancer Res 18: 5160-5162, 2012.

11 Francone TD, Landmann RG, Chen CT, Sun MY, Kuntz EJ, Zeng Z, Dematteo RP, Paty PB and Weiser MR: Novel xenograft model expressing human hepatocyte growth factor shows liganddependent growth of c-Met-expressing tumors. Mol Cancer Ther 6: 1460-1466, 2007.

12 Isella C, Terrasi A, Bellomo SE, Petti C, Galatola G, Muratore A, Mellano A, Senetta R, Cassenti A, Sonetto C, Inghirami G, Trusolino L, Fekete Z, De Ridder M, Cassoni P, Storme G, Bertotti A and Medico E: Stromal contribution to the colorectal cancer transcriptome. Nat Genet 47: 312-319, 2015.

13 Pepke S, Wold B and Mortazavi A: Computation for ChIP-seq and RNA-seq studies. Nat Methods 6: S22-32, 2009.

14 Chemaitilly W, Merchant TE, Li Z, Barnes N, Armstrong GT, Ness KK, Pui CH, Kun LE, Robison LL, Hudson MM, Sklar CA and Gajjar A: Central precocious puberty following the diagnosis and treatment of paediatric cancer and central nervous system tumours: presentation and long-term outcomes. Clin Endocrinol (Oxf) 84: 361-371, 2016.

15 Dobin A, Davis CA, Schlesinger F, Drenkow J, Zaleski C, Jha S, Batut P, Chaisson $M$ and Gingeras TR: STAR: ultrafast universal RNA-seq aligner. Bioinformatics 29: 15-21, 2013. 
16 Raskatov JA, Nickols NG, Hargrove AE, Marinov GK, Wold B and Dervan PB: Gene expression changes in a tumor xenograft by a pyrrole-imidazole polyamide. Proc Natl Acad Sci USA 109: 16041-16045, 2012.

17 Liao Y, Smyth GK and Shi W: The Subread aligner: fast, accurate and scalable read mapping by seed-and-vote. Nucleic Acids Res 41: e108, 2013.

18 Mootha VK, Lindgren CM, Eriksson KF, Subramanian A, Sihag S, Lehar J, Puigserver P, Carlsson E, Ridderstrale M, Laurila E, Houstis N, Daly MJ, Patterson N, Mesirov JP, Golub TR, Tamayo P, Spiegelman B, Lander ES, Hirschhorn JN, Altshuler D and Groop LC: PGC-1alpha-responsive genes involved in oxidative phosphorylation are coordinately downregulated in human diabetes. Nat Genet 34: 267-273, 2003.

19 Subramanian A, Tamayo P, Mootha VK, Mukherjee S, Ebert BL, Gillette MA, Paulovich A, Pomeroy SL, Golub TR, Lander ES and Mesirov JP: Gene set enrichment analysis: a knowledgebased approach for interpreting genome-wide expression profiles. Proc Natl Acad Sci USA 102: 15545-15550, 2005.

20 O'Connell MR, Sarkar S, Luthra GK, Okugawa Y, Toiyama Y, Gajjar AH, Qiu S, Goel A and Singh P: Epigenetic changes and alternate promoter usage by human colon cancers for expressing DCLK1-isoforms: Clinical Implications. Sci Rep 5: 14983, 2015.

21 Yoshihara K, Shahmoradgoli M, Martinez E, Vegesna R, Kim H, Torres-Garcia W, Trevino V, Shen H, Laird PW, Levine DA, Carter SL, Getz G, Stemke-Hale K, Mills GB and Verhaak RG: Inferring tumour purity and stromal and immune cell admixture from expression data. Nat Commun 4: 2612, 2013.
22 Warnes G, Bolker B, Bonebakker L, Gentleman R, Huber W, Liaw A, Lumley T, Maechler M, Magnusson A, Moeller S, Schwartz M and Venables B: gplots: Various R programming tools for plotting data: In: R package version. 2: 4, 2009.

23 Hidalgo M, Amant F, Biankin AV, Budinska E, Byrne AT, Caldas C, Clarke RB, de Jong S, Jonkers J, Maelandsmo GM, RomanRoman S, Seoane J, Trusolino L and Villanueva A: Patientderived xenograft models: an emerging platform for translational cancer research. Cancer Discov 4: 998-1013, 2014.

24 Quail DF and Joyce JA: Microenvironmental regulation of tumor progression and metastasis. Nat Med 19: 1423-1437, 2013.

25 Chou J, Fitzgibbon MP, Mortales CL, Towlerton AM, Upton MP, Yeung RS, McIntosh MW and Warren EH: Phenotypic and transcriptional fidelity of patient-derived colon cancer xenografts in immune-deficient mice. PLoS One 8: e79874, 2013.

26 Hylander BL, Punt N, Tang H, Hillman J, Vaughan M, Bshara W, Pitoniak R and Repasky EA: Origin of the vasculature supporting growth of primary patient tumor xenografts. J Transl Med 11: 110, 2013. 